Cytopathology 2020 (DOI: 10.1111/cyt.12921)

\title{
The phosphatase PTPN1/PTP1B is a candidate marker of better \\ chemotherapy response in metastatic high-grade serous carcinoma
}

Running title: PTPN1 in serous effusions

Ben Davidson, MD PhD ${ }^{1,2}$, Annika Jøntvedt Bock, $\mathrm{MD}^{1 *}$, Arild Holth, BSc${ }^{1}$, Dag Andre

Nymoen, $\mathrm{PhD}^{1 * *}$

${ }^{1}$ Department of Pathology, Oslo University Hospital, Norwegian Radium Hospital, N-0310 Oslo, Norway; ${ }^{2}$ University of Oslo, Faculty of Medicine, Institute of Clinical Medicine, N-0316 Oslo,

Norway

*Current address: Department of Anesthesiology, Telemark Hospital Trust, N-3710 Skien,

Norway

**Current address: Department of Medical Genetics, Oslo University Hospital, Norwegian

Radium Hospital, N-0310, Oslo, Norway

Word count (text): 2,471

Tables: 3

Figures: 2

\section{$\underline{\text { Acknowledgements }}$}

Conflict of interest: none

Funding: This work was supported by The Inger and John Fredriksen Foundation for Ovarian

Cancer Research.

Data availability statement: Research data are not shared 


\section{Corresponding author}

Ben Davidson, MD PhD

Department of Pathology

Norwegian Radium Hospital

Oslo University Hospital

Montebello N-0310 Oslo

Norway

Tel: (47) 22782415

Email: bend@medisin.uio.no 


\begin{abstract}
$\underline{\text { Abstract }}$
Objective: To analyze the expression and clinical role of the phosphatase PTPN1 (PTP1B) in serous effusions.
\end{abstract}

Methods: PTPN1 mRNA expression by quantitative RT-PCR was analyzed in 83 high-grade serous carcinoma (HGSC) and 15 malignant mesothelioma (MM) effusions. PTP1B and phospho-PTP1B (pPTP1B) protein expression by immunohistochemistry was analyzed in 62 HGSC and 44 MM effusions.

Results: PTPN1 mRNA ( $\mathrm{p}=0.048)$, PTP1B protein $(\mathrm{p}=0.047)$ and $\mathrm{pPTP1B}$ protein $(\mathrm{p}<0.001)$ were overexpressed in HGSC compared to MM effusions. PTPN1 mRNA was additionally overexpressed in post-chemotherapy HGSC effusions compared to chemo-naïve effusions $(p=0.005)$. However, $p$ PTP1B protein expression was higher in effusions from patients with FIGO stage III compared to stage IV disease $(\mathrm{p}=0.006)$, and higher expression of both PTPN1 mRNA $(p=0.041)$ and PTP1B protein $(p=0.035)$ in HGSC effusions was associated with better (complete) chemotherapy response at diagnosis. PTPN1 RNA and protein expression was unrelated to survival in HGSC, whereas a trend for shorter overall survival $(p=0.06)$ was found for MM patients whose tumors expressed pPTP1B protein.

Conclusion: PTPN1 is overexpressed in HGSC compared to MM effusions, and may be a marker of better chemotherapy response in the former. Whether PTPN1 activation is informative of adverse outcome in MM merits further investigation.

Keywords: PTPN1; high-grade serous carcinoma; malignant mesothelioma; effusion 


\section{Introduction}

Proteins phosphatases, like protein kinases, are major regulators of cell signaling, and through it of the biology of normal cells. As protein kinases, their expression and function are dysregulated in cancer, often through mutation and deactivation of tumor-suppressing phosphatases, e.g. phosphatase and tensin homologue located on chromosome 10 (PTEN), which is the $2^{\text {nd }}$ most commonly mutated tumor suppressor cancer, after TP53. Proteins phosphatases, numbering about 150, consist of two families, protein serine/threonine phosphatases (PSP) and protein tyrosine phosphatases (PTP). PTPs are in turn divided into Cys-based, Asp-based and His-based subclasses, the former further divided into three classes (I-III). Class I of the Cys-based PTPs is still further divided into 6 subclasses labelled I-VI [1].

PTPN1 (PTP1B) is a classical non-receptor phosphatase belonging to subclass I within the class I cys-based PTP group. The PTPN1 gene is localized to the q13.1-q13.2 area of chromosome 20, a region that is gained or amplified in several cancers. The PTPN1 promoter area binds several transcription factors, including YB-1, NF-אB, EGR-1, SP1 and SP3. PTPN1 substrates include receptor protein kinases, intracellular protein tyrosine kinases, adapter proteins, cytoskeletal proteins and transcription factors, with a resulting effect on proliferation, differentiation, glucose metabolism, apoptosis, adhesion, motility and invasion. The net effect of PTPN1 activity may be tumor-promoting or tumor-inhibiting depending on its targets in a given specific tumor [2]

Ovarian cancer, consisting predominantly of ovarian carcinoma, $(\mathrm{OC})$ is the $8^{\text {th }}$ most common and $8^{\text {th }}$ most lethal cancer in women globally, with 295,414 new cases and 184,799 deaths in 2018 [3]. The most common ovarian carcinoma histotype, high-grade serous carcinoma (HGSC), has its origin in the fallopian tube rather than the ovary in the majority of cases, and is 
characterized by aggressive clinical course due to diagnosis at advanced stage and increasing chemoresistance along disease progression $[4,5]$.

Two previous studies of OC have shown amplification of chromosome 20q, harboring the PTPN1 gene $[6,7]$. The protein product of this gene, PTP1B, was reported to be overexpressed in OC compared to normal ovarian epithelium in one study [8], though no overexpression was seen in comparison with benign and borderline tumors in another study [9]. PTPN1 has been additionally reported to have a role in cellular signaling and to regulate migration, invasion, metastasis, proliferation and cell survival in experimental OC models [10-15]. Data regarding the prognostic role of this molecule is to date limited to a single study, in which high PTPIB mRNA levels were associated with poor overall survival (OS) in analysis of a public database containing 468 tumors of unspecified histotype [15].

The objective of the present study was to quantitatively analyze the expression and clinical role of PTPN1 at the mRNA and protein level in HGSC effusions. Malignant mesothelioma (MM), a tumor with a metastatic pattern similar to HGSC, was studied for comparative purposes. 


\section{Material and Methods}

\subsection{Patients and specimens}

Quantitative RT-PCR (qRT-PCR) cohort: Effusions consisted of 83 HGSC specimens (63 peritoneal, 20 pleural) submitted to the Department of Pathology at the Norwegian Radium Hospital during the period of 1998 to 2004 . A series of 15 MM effusions (4 peritoneal, 11 pleural) submitted to the Departments of Pathology at the Norwegian Radium Hospital and Aalborg University Hospital, Aalborg, Denmark, during the period of 1998 to 2007 were studied for comparative purposes. The latter were studied with kind permission from Mr. Søren Nielsen (Department of Pathology, Aalborg University Hospital). MM effusions were from patients diagnosed with epithelioid or biphasic MM in biopsy specimens, and cells in the effusion specimens had epithelioid morphology. Clinicopathologic data for the HGSC cohort are detailed in Table 1.

Effusions were centrifuged immediately after tapping, and cell pellets were frozen at $-70^{\circ} \mathrm{C}$ in equal amounts of RPMI 1640 medium (GIBCO-Invitrogen, Carlsbad, CA) containing 50\% fetal calf serum (PAA Laboratories GmbH, Pasching, Austria) and 20\% dimethylsulfoxide (Merck KGaA, Darmstadt, Germany). Effusion specimens were diagnosed by an experienced cytopathologist (BD) based on morphology in smears and cell blocks, prepared using the thrombin clot protocol, and IHC, based on established guidelines [16].

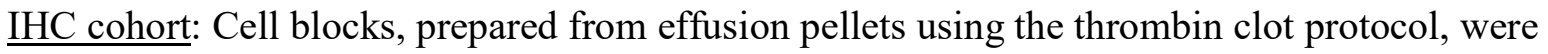
available for 62 of the 83 patients with HGSC effusions (45 peritoneal, 17 pleural). Additionally, $44 \mathrm{MM}$ effusions (14 peritoneal, 30 pleural), which included cell blocks from the 15 specimens analyzed using qRT-PCR, were studied. MM effusions were submitted to the Departments of Pathology at the Norwegian Radium Hospital and Aalborg University Hospital 
during the period of 1999 to 2008, and were from 37 patients (21 males, 16 females; 31 with one specimen, 5 with 2 specimens, 1 with 3 specimens).

Informed consent was obtained according to national and institutional guidelines. Study approval was given by the Regional Committee for Medical Research Ethics in Norway (S-04300).

\section{2. qRT-PCR}

Effusions were centrifuged to obtain a cell pellet from which RNA was extracted using QIAsymphony (Qiagen, Hilden Germany). Details regarding reverse transcription, primer and probe design procedure and software, and efficiency testing were previously described [17]. The qRT-PCR reaction was run using the Perfecta qPCR ToughMix (Quanta Biosciences, Gaithersburg MD) and quantified on the Roche LightCycler 480 (Roche, Basel, Switzerland). Samples were analyzed in triplicate and average copy number was used for statistical analysis. PTPN1 primer and probe sequences were as follows:

Forward: TGATACCTGCCTCTTGCTGATG

Reverse: CCCATCCGAAACTTCCTCAT

Probe: CAAGAGGAAAGACCCTTCTTCCGTTGATATCAAGA

The beta-glucuronidase (GUS), TATA box binding protein $(T B P)$ and mitochondrial ribosomal protein L19 (MRLP19) genes were used as reference genes following previous testing [17] applying established guidelines [18-20]. Primer and probe sequences were previously detailed [17].

\subsection{IHC}

Formalin-fixed, paraffin-embedded sections from $62 \mathrm{HGSC}$ and $44 \mathrm{MM}$ effusions were analyzed for PTP1B and phospho-PTP1B S378 (pPTP1B) protein expression using the Dako EnVision 
Flex + System (K8012; Dako, Glostrup, Denmark). The PTP1B antibody was a mouse monoclonal antibody purchased from Abcam (cat \# ab201974, clone 4F8F11; Cambridge, UK), applied at a 1:400 dilution following antigen retrieval in Low $\mathrm{pH}$ buffer $(\mathrm{pH}$ 6.0). The pPTP1B antibody was a rabbit monoclonal antibody purchased from Abcam (cat \# ab76239, clone EP1840Y), applied at a 1:50 dilution following antigen retrieval in Low $\mathrm{pH}$ buffer ( $\mathrm{pH}$ 6.0). Following deparaffinization and antigen retrieval, sections were treated with EnVision ${ }^{\mathrm{TM}}$ Flex + mouse linker (15 min) and EnVision ${ }^{\mathrm{TM}}$ Flex/HRP enzyme (30 minutes) and stained for 10 minutes with 3'3-diaminobenzidine tetrahydrochloride (DAB), counterstained with hematoxylin, dehydrated and mounted in Toluene-Free Mounting Medium (Dako). Positive controls for PTP1B and pPTP1B consisted of breast carcinoma and seminoma, respectively. In negative controls, the primary antibody was replaced with isotype-specific mouse myeloma protein diluted to the same concentration as the primary antibody for PTP1B, and with isotype-specific rabbit protein for pPTP1B.

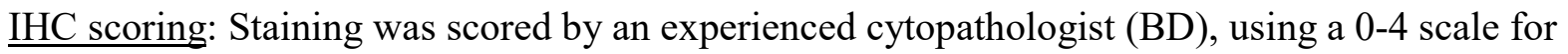
staining extent as follows: $0=$ no staining, $1=1-5 \%, 2=6-25 \%, 3=26-75 \%, 4=76-100 \%$ of tumor cells. Staining intensity was scored as 0,1 or 2 , corresponding to negative, weak or strong staining. Values were combined by doubling, providing a 0-8 score.

\subsection{Statistical analysis}

Statistical analysis was performed applying the SPSS-PC package (Version 26). Probability of $<0.05$ was considered statistically significant. The Mann-Whitney U test or the Kruskal-Wallis H test was applied to analysis of the association between PTPN1 mRNA and PTP1B/pPTP1B protein expression by IHC and clinicopathologic parameters (for 2-tier or 3-tier analyses, respectively). For this analysis, clinicopathologic parameters were grouped as follows: age: $\leq 60$ 
vs. $>60$ years; effusion site: peritoneal vs. pleural; FIGO stage: III vs. IV; chemotherapy status: pre- vs. post-chemotherapy specimens; residual disease (RD) volume: $0 \mathrm{~cm}$ vs. $\leq 1 \mathrm{~cm}$ vs. $>1 \mathrm{~cm}$; response to chemotherapy: complete response vs. partial response/stable disease/progressive disease. Platinum resistance was defined as PFS $\leq 6$ months according to guidelines published by the Gynecologic Oncology Group (GOG) and progressive disease or recurrence was evaluated by the Response Evaluation Criteria In Solid Tumors (RECIST) criteria.

Progression-free survival (PFS) and OS were calculated from the date of the last chemotherapy treatment/diagnosis to the date of recurrence/death or last follow-up, respectively. Univariate survival analyses of PFS and OS were executed using the Kaplan-Meier method and log-rank test. For this analysis in the HGSC cohort, mRNA values were divided to high or low based on median expression, whereas for protein expression, staining score of 0-4 was grouped as low, score $>4$ as high. 


\section{Results}

Comparative analysis of PTPN1 expression by qRT-PCR in 83 HGSC and 15 MM effusions showed higher levels of this mRNA in the former group, though with marginal significance $($ mean rank $=51.92 \mathrm{vs}, 36.13 ; \mathrm{p}=0.048)$.

IHC analysis showed cytoplasmic expression of PTP1B, whereas pPTP1B was expressed in both the cytoplasm and nucleus of HGSC and MM cells (Figure 1). Cytoplasmic staining differed in intensity and was consequently scored using a combined value for extent and intensity, as detailed in the Materials and methods section, whereas nuclear staining was of comparable intensity and therefore scored only for extent (Table 2).

In agreement with the mRNA finding, cytoplasmic PTP1B and pPTP1B expression by IHC was significantly higher in the 62 HGSC analyzed compared to $44 \mathrm{MM}$ effusions ( $p=0.047$ and $\mathrm{p}<0.001$ for PTP1B and $\mathrm{pPTP} 1 \mathrm{~B}$, respectively). No difference was observed for nuclear pPTP1B expression $(\mathrm{p}=0.828)$.

The association between PTPN1 expression at the mRNA and protein level was assessed. In HGSC, PTPN1 mRNA levels were significantly higher in post-chemotherapy effusions compared to pre-chemotherapy specimens tapped at diagnosis (mean rank $=49.38$ vs. 34.44 ; $\mathrm{p}=0.005)$. PTPN1 mRNA levels were additionally higher in specimens from patients who had favorable (complete) response to chemotherapy at diagnosis compared to specimens from patients who had unfavorable response (partial response, stable disease or progression; mean rank $=40.19$ vs. $30.24 ; p=0.041)$.

In analysis of IHC results, higher cytoplasmic pPTP1B expression score was observed in effusions from patients diagnosed with FIGO stage III compared to stage IV disease ( $\mathrm{p}=0.006$;

Table 3), whereas PTP1B cytoplasmic score was higher in effusions from patients with better 
chemotherapy response at diagnosis and was inversely related to intrinsic chemoresistance $(\mathrm{p}=0.035$ and $\mathrm{p}=0.036$, respectively; Table 3$)$.

Survival data were available for all HGSC patients in the qRT-PCR cohort, the follow-up period ranged from 1 to 179 months (mean $=33$ months, median $=24$ months). PFS ranged from 0 to 148 months (mean $=9$ months, median $=5$ months). At the last follow-up, 81 patients were dead of disease, 1 was alive with disease and 1 was with no evidence of disease. PTPN1 mRNA levels were unrelated to OS $(\mathrm{p}=0.646)$ or PFS $(\mathrm{p}=0.541)$ in univariate survival analysis, with comparable data in separate analysis of patients with pre- and post-chemotherapy specimens ( $p>0.05$; data not shown). Analysis of the protein expression data similarly showed no association with $\mathrm{OS}(\mathrm{p}=0.216, \mathrm{p}=0.211$ and $\mathrm{p}=0.41$ for cytoplasmic $\mathrm{PTP} 1 \mathrm{~B}$, cytoplasmic $\mathrm{pPTP} 1 \mathrm{~B}$ and nuclear pPTP1B, respectively) or with PFS, though a trend for longer PFS was observed for cytoplasmic PTP1B $(\mathrm{p}=0.076, \mathrm{p}=0.502$ and $\mathrm{p}=0.995$ for cytoplasmic PTP1B, cytoplasmic pPTP1B and nuclear pPTP1B, respectively; Figure 2-A).

In analysis of clinical parameters in the MM IHC cohort, performed for all 44 specimens, PTP1B score was higher in specimens from male patients compared to effusions from female patients (mean rank $=26.52$ vs. $18.10 ; p=0.023$ ) and in pleural compared to peritoneal effusions (mean rank $=25.50$ vs. $16.07 ; p=0.018)$. Cytoplasmic and nuclear $p$ PTP1B expression did not differ significantly between these groups, whereas none of the 3 IHC parameters was related to patient age (cut-off at 70 years; $p>0.05$ ).

Survival data were available for 29 of the $37 \mathrm{MM}$ patients in the IHC cohort, all treated at Oslo University Hospital. In this cohort, the follow-up period ranged from 2 to 141 months (mean $=30$ months, median $=18$ months). At the last follow-up, 28 patients were dead of disease and 1 patient was lost to follow-up. PTP1B and pPTP1B expression was not significantly related to OS, 
but a trend for shorter survival was observed for patients with tumors expressing cytoplasmic pPTP1B expression compared to those with negative ones $(\mathrm{p}=0.122, \mathrm{p}=0.06$ and $\mathrm{p}=0.637$ for cytoplasmic PTP1B, cytoplasmic pPTP1B and nuclear pPTP1B, respectively; Figure 2-B), 


\section{Discussion}

PTP1B protein expression in OC has been documented in 2 studies performed in the 1990's $[8,9]$. However, both studies were small and have analyzed tumors of various histotype, a practice which was common prior to the 2014 WHO book. The present study is the first to analyze a wellcharacterized cohort limited to HGSC, focusing on metastatic tumor cells in effusions, and documents frequent expression of PTPN1 mRNA and PTP1B protein, as well as its activated form, $\mathrm{pPTP} 1 \mathrm{~B}$, in this malignancy.

PTPN1 expression in HGSC was compared to its presence in MM, a tumor which shares the propensity to involve the serosal cavities displayed by HGSC, as well as some of the morphological, immunohistochemical and genetic features of the latter cancer. In MM, 2 previous reports have implicated PTP1B in modulation of PI3K signaling in response to the presence of free fatty acids [21] and in mediation of apoptosis following exposure to Dipalmitoleoyl-phosphatidylethanolamine (DPPE) [22], but no data is available regarding expression of this molecule in clinical specimens. Differences between HGSC and MM were moderate, though statistically significant, for PTPN1 mRNA and PTP1B protein expression, and were more clearly evident for pPTPN1 at its cytoplasmic, but not nuclear localization. The molecular partners of pPTPN1 in these 2 compartments have not been characterized to the best of our knowledge.

Bartolomé et al. recently reported an association between $P T P 1 B$ mRNA levels and poor OS in $\mathrm{OC}$ in an in silico analysis of the GEPIA2 dataset, but the histology of the tumors analyzed was not specified, rendering it difficult to draw conclusions from this study [15].

Studies of other cancers have generated variable results. Low PTP1B expression was reported to be associated with poor survival in hepatocellular carcinoma [23], while the opposite was found 
in colorectal carcinoma $[24,25]$. Two studies of breast carcinoma have generated opposite results with respect to prognosis [26,27], whereas association with poor outcome was found in a recent study of neuroblastoma [28].

In the present study, PTPN1 mRNA levels were significantly higher in post-chemotherapy effusions, the majority obtained at disease recurrence, compared to chemo-naïve specimens tapped at diagnosis, suggesting, despite the fact the specimens were not patient-matched, an association with disease progression. However, higher PTPN1 mRNA levels, as well as higher cytoplasmic PTP1B protein expression, were significantly associated with favorable chemoresponse at diagnosis, a finding which suggests a tumor-suppressing role for this molecule in HGSC. The absence of significant association with poor survival, with a trend for longer PFS, further argues against a role for PTPN1 in promoting aggressiveness and progression in this cancer. In contrast, the finding of a trend towards shorter survival in MM may merit analysis of a larger cohort in order to further assess its clinical role.

In conclusion, PTPN1 mRNA, PTP1B and pPTP1B are frequently expressed in HGSC and MM effusions, possibly with opposing clinical roles. Identifying the molecular partners in each tumor in the different sub-cellular components is necessary for better defining the biological role of PTPN1 in both cancers. 


\section{$\underline{\text { References }}$}

1. Meeusen B, Janssens V. Tumor suppressive protein phosphatases in human cancer: Emerging targets for therapeutic intervention and tumor stratification. Int J Biochem Cell Biol. 2018;96:98134.

2. Lessard L, Stuible M, Tremblay ML. The two faces of PTP1B in cancer. Biochim Biophys Acta. 2010;1804(3):613-619.

3. Bray F, Ferlay J, Soerjomataram I, Siegel RL, Torre LA, Jemal A. Global cancer statistics 2018: GLOBOCAN estimates of incidence and mortality worldwide for 36 cancers in 185 countries. CA Cancer J Clin. 2018;68(6):394-424.

4. Singh N, McCluggage WG, Gilks CB. High-grade serous carcinoma of tubo-ovarian origin: recent developments. Histopathology. 2017;71(3):339-356.

5. Lheureux S, Braunstein M, Oza AM. Epithelial ovarian cancer: Evolution of management in the era of precision medicine. CA Cancer J Clin. 2019;69(4):280-304.

6. Watanabe T, Imoto I, Katahira T, et al. Differentially regulated genes as putative targets of amplifications at 20q in ovarian cancers. Jpn J Cancer Res. 2002;93(10):1114-1122.

7. Tanner MM, Grenman S, Koul A, et al. Frequent amplification of chromosomal region 20q12q13 in ovarian cancer. Clin Cancer Res. 2000;6(5):1833-1839.

8. Wiener JR, Hurteau JA, Kerns BJ, et al. Overexpression of the tyrosine phosphatase PTP1B is associated with human ovarian carcinomas. Am J Obstet Gynecol. 1994;170(4):1177-1183.

9. van Haaften-Day C, Russell P, Boyer CM, et al. Expression of cell regulatory proteins in ovarian borderline tumors. Cancer. 1996;77(10):2092-2098.

10. Huang RY, Wen CC, Liao CK, Wang SH, Chou LY, Wu JC. Lysophosphatidic acid modulates the association of PTP1B with $\mathrm{N}$-cadherin/catenin complex in SKOV3 ovarian cancer cells. Cell Biol Int. 2012;36(9):833-841. 
11. Fan G, Lin G, Lucito R, Tonks NK. Protein-tyrosine phosphatase $1 \mathrm{~B}$ antagonized signaling by insulin-like growth factor-1 receptor and kinase BRK/PTK6 in ovarian cancer cells. $J$ Biol Chem. 2013;288(34):24923-24934.

12. Shahzad MMK, Felder M, Ludwig K, et al. Trans10,cis 12 conjugated linoleic acid inhibits proliferation and migration of ovarian cancer cells by inducing ER stress, autophagy, and modulation of Src. PLoS One. 2018;13(1):e0189524.

13. Zhang J, Wang Z, Zhang S, et al. Spatial regulation of signaling by the coordinated action of the protein tyrosine kinases MET and FER. Cell Signal. 2018;50:100-110.

14. Wang W, Cao Y, Zhou X, Wei B, Zhang Y, Liu X. PTP1B promotes the malignancy of ovarian cancer cells in a JNK-dependent mechanism. Biochem Biophys Res Commun. 2018;503(2):903-909.

15. Bartolomé RA, Martín-Regalado Á, Jaén M, et al. Protein Tyrosine Phosphatase-1B Inhibition Disrupts IL13Ra2-Promoted Invasion and Metastasis in Cancer Cells. Cancers (Basel). 2020;12(2):500.

16. Davidson B, Firat P, Michael CM (eds.): Serous effusions - etiology, diagnosis, prognosis and therapy. Springer, London, $2^{\text {nd }}$ ed., 2018, p. 49-74.

17. Hetland TE, Nymoen DA, Emilsen E, et al. MGST1 expression in serous ovarian carcinoma differs at various anatomic sites, but is unrelated to chemoresistance or survival. Gynecol Oncol. 2012;126(3):460-465.

18. Bustin SA, Benes V, Garson JA, et al. The MIQE Guidelines: Minimum Information for Publication of Quantitative Real-Time PCR Experiments. Clin Chem. 2009;55(4):611-622. 19. Andersen CL, Jensen JL, Orntoft T. Normalization of real-time quantitative reverse transcription-PCR data: a model-based variance estimation approach to identify genes suited for 
normalization, applied to bladder and colon cancer data sets. Cancer Res. 2004;64(15):52455250.

20. Vandesompele J, De Preter K, Pattyn F, et al. Accurate normalization of real-time quantitative RT-PCR data by geometric averaging of multiple internal control genes. Genome Biol. 2002;3(7):RESEARCH0034.

21. Shibata E, Kanno T, Tsuchiya A, et al. Free fatty acids inhibit protein tyrosine phosphatase 1B and activate Akt. Cell Physiol Biochem. 2013;32(4):871-879.

22. Kaku Y, Tsuchiya A, Kanno T, Nakano T, Nishizaki T. Dipalmitoleoylphosphatidylethanolamine induces apoptosis of NCI-H28 malignant mesothelioma cells. Anticancer Res. 2014;34(4):1759-1764.

23. Zheng LY, Zhou DX, Lu J, Zhang WJ, Zou DJ. Down-regulated expression of the proteintyrosine phosphatase 1B (PTP1B) is associated with aggressive clinicopathologic features and poor prognosis in hepatocellular carcinoma. Biochem Biophys Res Commun. 2012;420(3):680684.

24. Chen Q, Li Y, Li Z, Zhao Q, Fan L. Overexpression of PTP1B in human colorectal cancer and its association with tumor progression and prognosis. J Mol Histol. 2014;45(2):153-159. 25. Hoekstra E, Das AM, Swets M, et al. Increased PTP1B expression and phosphatase activity in colorectal cancer results in a more invasive phenotype and worse patient outcome. Oncotarget. 2016;7(16):21922-21938.

26. Soysal S, Obermann EC, Gao F, et al. PTP1B expression is an independent positive prognostic factor in human breast cancer. Breast Cancer Res Treat. 2013;137(2):637-644. 27. Liu X, Chen Q, Hu XG, et al. PTP1B promotes aggressiveness of breast cancer cells by regulating PTEN but not EMT. Tumour Biol. 2016;37(10):13479-13487. 
28. Nunes-Xavier CE, Aurtenetxe O, Zaldumbide L, et al. Protein tyrosine phosphatase PTPN1 modulates cell growth and associates with poor outcome in human neuroblastoma. Diagn Pathol. 2019;14(1):134. 
Table 1: Clinicopathologic parameters of the HGSC qRT-PCR cohort $(n=83)$

\begin{tabular}{|c|c|c|}
\hline \multicolumn{2}{|l|}{ Parameter } & No. of cases \\
\hline \multicolumn{2}{|l|}{ Age (mean) } & $35-87$ years $(62)$ \\
\hline \multicolumn{2}{|l|}{ FIGO stage } & \\
\hline \multicolumn{2}{|l|}{$\mathrm{I}$} & 0 \\
\hline \multicolumn{2}{|l|}{ II } & 1 \\
\hline \multicolumn{2}{|l|}{ III } & 44 \\
\hline \multicolumn{2}{|l|}{ IV } & 38 \\
\hline \multicolumn{2}{|l|}{ Residual disease } & \\
\hline \multirow{2}{*}{$\begin{array}{l}\text { Primary debulking surgery }(\mathrm{n}=51 \text { and } \\
\mathrm{n}=140 \text { for the qRT-PCR and IHC }\end{array}$} & 0 & 2 \\
\hline & $\leq 1 \mathrm{~cm}$ & 20 \\
\hline cohort, respectively) & $>1 \mathrm{~cm}$ & 29 \\
\hline \multirow{2}{*}{$\begin{array}{l}\text { Interval debulking surgery }(\mathrm{n}=18 \text { and } \\
\mathrm{n}=77 \text { for the } \mathrm{qRT}-\mathrm{PCR} \text { and IHC cohort, }\end{array}$} & 0 & 3 \\
\hline & $\leq 1 \mathrm{~cm}$ & 6 \\
\hline respectively) & $>1 \mathrm{~cm}$ & 9 \\
\hline \multicolumn{2}{|l|}{ NA } & 14 \\
\hline \multicolumn{2}{|c|}{ CA 125 at diagnosis (range; median) ${ }^{a}$} & $10-43800(1474)$ \\
\hline \multicolumn{3}{|c|}{ Chemoresponse after primary treatment } \\
\hline $\mathrm{CR}$ & & 37 \\
\hline PR & & 13 \\
\hline $\mathrm{SD}$ & & 3 \\
\hline PD & & 17 \\
\hline $\mathrm{NA}^{b}$ & & 13 \\
\hline
\end{tabular}


Abbreviations: $\mathrm{NA}=$ not available; $\mathrm{CR}=$ complete response; $\mathrm{PR}=$ partial response; $\mathrm{SD}=$ stable disease $; \mathrm{PD}=$ progressive disease

${ }^{a}$ Available for 55 patients

${ }^{b}$ Not available (missing data or disease response after chemotherapy could not be evaluated because of normalized CA 125 after primary surgery or missing CA 125 information and no residual tumor). 
Table 2: PTP1B and pPTP1B protein expression in HGSC $(n=62)$ and MM $(n=44)$ effusions

\begin{tabular}{|c|c|c|c|c|c|c|c|c|c|}
\hline \multirow[t]{2}{*}{ Diagnosis } & \multirow[t]{2}{*}{ Protein } & \multirow[t]{2}{*}{ Localization } & \multicolumn{7}{|c|}{ Staining score (extent $x$ intensity) ${ }^{a}$} \\
\hline & & & 0 & 1 & 2 & 3 & 4 & 6 & 8 \\
\hline HGSC & PTP1B & Cytoplasm & 0 & 1 & 2 & 8 & 12 & 0 & 39 \\
\hline HGSC & pPTP1B & Cytoplasm & 7 & 9 & 9 & 11 & 11 & 2 & 13 \\
\hline MM & PTP1B & Cytoplasm & 3 & 3 & 2 & 5 & 7 & 5 & 19 \\
\hline MM & pPTP1B & Cytoplasm & 22 & 10 & 4 & 3 & 3 & 1 & 1 \\
\hline \multirow[t]{2}{*}{ Diagnosis } & Protein & Localization & \multicolumn{7}{|c|}{ Staining extent (\% of cells) } \\
\hline & & & $0 \%$ & \multicolumn{2}{|l|}{$1-5 \%$} & $6-25 \%$ & \multicolumn{2}{|c|}{$26-75 \%$} & $76-100 \%$ \\
\hline HGSC & pPTP1B & Nucleus & 14 & \multicolumn{2}{|l|}{27} & 10 & \multicolumn{2}{|l|}{10} & 1 \\
\hline MM & pPTP1B & Nucleus & 13 & \multicolumn{2}{|l|}{13} & 6 & \multicolumn{2}{|l|}{12} & 0 \\
\hline
\end{tabular}

Abbreviations: $\mathrm{HGSC}=$ high-grade serous carcinoma; $\mathrm{MM}=$ malignant mesothelioma

${ }^{a}$ Significantly higher cytoplasmic PTP1B $(\mathrm{p}=0.047)$ and $\mathrm{pPTP} 1 \mathrm{~B}(\mathrm{p}<0.001)$ expression in HGSC effusions. 
Table 3: Significant association between PTP1B/pPTP1B expression in HGSC effusions and clinicopathologic parameters

\begin{tabular}{|c|c|c|c|c|c|c|c|c|}
\hline \multirow[t]{2}{*}{ FIGO stage ${ }^{a}$} & \multicolumn{7}{|c|}{ Cytoplasmic pPTP1B staining score (extent $x$ intensity) $^{a}$} & \multirow[t]{2}{*}{ p-value } \\
\hline & 0 & 1 & 2 & 3 & 4 & 6 & 8 & \\
\hline III $(n=32)$ & 2 & 2 & 3 & 7 & 7 & 2 & 9 & $\mathrm{p}=0.006$ \\
\hline IV $(n=29)$ & 5 & 7 & 5 & 4 & 4 & 0 & 4 & \\
\hline Chemotherapy & \multicolumn{7}{|c|}{ Cytoplasmic PTP1B staining score (extent $x$ intensity) $^{a}$} & p-value \\
\hline response $^{b}$ & 0 & 1 & 2 & 3 & 4 & 6 & 8 & \\
\hline Complete $(\mathrm{n}=28)$ & 0 & 0 & 0 & 2 & 4 & 0 & 22 & \multirow[t]{2}{*}{$\mathrm{p}=0.035$} \\
\hline Other $(n=23)^{c}$ & 0 & 1 & 0 & 5 & 5 & 0 & 12 & \\
\hline Primary & \multicolumn{7}{|c|}{ 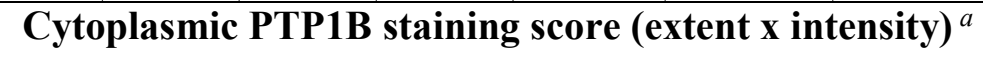 } & p-value \\
\hline chemoresistance $^{d}$ & 0 & 1 & 2 & 3 & 4 & 6 & 8 & \\
\hline No $(n=27)$ & 0 & 0 & 0 & 3 & 3 & 0 & 21 & \multirow[t]{2}{*}{$\mathrm{p}=0.036$} \\
\hline Yes $(n=35)$ & 0 & 1 & 2 & 5 & 9 & 0 & 18 & \\
\hline
\end{tabular}

${ }^{a}$ One patient with stage II disease excluded from the analysis.

${ }^{b}$ Eleven patients with missing data.

${ }^{c}$ Partial response, stable disease or progression

${ }^{d}$ PFS $\leq 6$ months 


\section{Figure legends}

\section{Figure 1: PTP1B and pPTP1B Immunohistochemistry}

(A-F) HGSC effusions. Strong (A) and predominantly weak (B) PTP1B staining; Strong (C) and weak (D) cytoplasmic pPTP1B staining. Few positive nuclei are additionally seen in (C); (E) Combined nuclear and weak cytoplasmic pPTP1B expression in some tumor cells; (F) Nuclear expression with no cytoplasmic staining.

(G-L) MM effusions. Strong (G), weak (H) and negative (I) PTP1B staining; Strong (J) and weak (K) cytoplasmic pPTP1B staining; (L) Focal $(<5 \%)$ nuclear and cytoplasmic pPTP1B expression.

\section{Figure 2: Survival}

A: Kaplan-Meier survival curve showing the association between PTP1B protein expression and progression-free survival (PFS) for 62 HGSC patients. Patients with effusions high (score $>4$ ) PTP1B expression ( $\mathrm{n}=39$; red line) had mean PFS of 12.4 months compared to 5.7 months for patients with effusions with low (score $\leq 4)$ expression $(n=23$, blue line; $p=0.076)$.

B: Kaplan-Meier survival curve showing the association between pPTP1B cytoplasmic expression and overall survival (OS) for $29 \mathrm{MM}$ patients. Patients with pPTP1B-positive effusions ( $\mathrm{n}=15$; red line) had mean OS of 19.7 months compared to 42.9 months for patients with pPTP1B-negative effusions ( $n=14$, blue line; $p=0.06)$. 